\title{
Dilemme en matière de prestation de
} soins

$\mathrm{Au}$ Canada, la politique sociale gouvernementale est fortement influencée par les tendances favorisant les compressions budgétaires. Sujet brûlant et délicat, le fardeau et la responsabilité des services sociaux et de santé sont passés du gouvernement fédéral aux provinces qui, à leur tour, les ont confiés à la communauté et aux familles. Les politiques provinciales présentent les soins aux personnes âgées comme une tâche incombant à la communauté et comptent sur la participation de la famille pour assurer la prestation des soins. Ces familles sont appuyées par des prestateurs de soins à domicile à très faible revenu. Les soins prodigués à la famille sont principalement assurés par les épouses et les filles, et les personnes rémunérées offrant des services de soins à domicile sont également et en règle générale des femmes. Par conséquent, ce sont les femmes qui, en matière de soins aux aînés, assument toujours les responsabilités sur les plans financier, affectif et physique dans le cadre de notre système de soins de santé.

Le problème que doivent surmonter les décideurs de politiques de soins de santé consiste à offrir des services de qualité aux personnes âgées de façon rentable et ce, sans exploiter les femmes. Malheureusement, la majorité des provinces n'ont pas encore trouvé la solution à ce problème. Premièrement, il est important de reconnaître qu'il existe bel et bien un problème. La prestation de soins, par des travailleurs rémunérés ou non, n'est pas considérée très importante au Canada. Cette tendance reflète le peu d'importance accordée aux besoins de service des aînés et aux personnes assurant ces services. En effet, la société s'attend à ce que les femmes fournissent des soins aux aînés moyennant une rémunération très modique ou même sans rétribution. Lors d'un entretien non officiel, un représentant provincial responsable des services de soins à long terme a déclaré que les familles devront continuer de fournir des soins aux membres âgés de leur famille car les gouvernements ne disposent pas des fonds nécessaires pour répondre à leurs besoins. Certains programmes de soins à domicile sont axés sur les services destinés aux aînés sans famille et offrent très peu d'appui aux personnes âgées vivant avec leur famille. Dans ces cas, on s'attend à ce que la conjointe ou la fille donne les soins requis.

La majorité des soins rémunérés offerts aux aînés sont assurés par des prestateurs de soins à domicile à faible revenu. Au sein des agences, ces personnes se trouvent au bas de l'échelle et disposent de très peu de moyens pour changer la structure du système. Dans bon nombre d'agences, ces travailleurs ne prennent pas part à la planification des services de soins, même si ce sont eux qui ont le plus de contacts avec la clientèle. De plus, ils reçoivent très peu d'information sur les clients des professionnels oeuvrant au sein du service. Même si la majorité des prestateurs de soins à domicile of- 
frent des services de qualité, ils ont peu d'occasions d'améliorer leurs connaissances ou compétences. En fait, la tendance en matière de prestation de soins aux aînés au sein de la communauté s'oriente vers des services offerts par des non-professionnels. La majorité des professions qui font actuellement l'objet de compressions budgétaires sont dominées par les femmes.

Dans le cadre d'un article publié dans cette revue, Sheila Neysmith et Barbara Nichols soulignent que les décideurs en matière de politiques se sont concentrés sur les tâches reliées à la prestation de soins et non sur les conditions de travail des prestateurs de soins. Cette approche fonctionnelle vis-à-vis des tâches donne naissance à des politiques qui ne tiennent compte ni des récipiendaires ni des prestateurs de soins. L'analyse de Neysmith et Nichols sur les conditions de travail des prestateurs de soins prodigués par des proches parents, des travailleurs à domicile et des bénévoles démontre clairement que la prestation de soins comporte beaucoup plus que des tâches fonctionnelles. Selon l'analyse, les conditions de travail en matière de soins fournis par les membres de la famille non rémunérés sont caractérisées par des difficultés sur le plan financier, un sentiment d'isolement, un manque d'information et de formation, une responsabilité de 24 heures et très peu ou aucun moment libre. Lorsque la prestation de soins à la famille est considérée sous l'optique d'un emploi avec de si mauvaises conditions de travail, les politiques actuelles, qui n'offrent aucune issue aux femmes, semblent très peu justifiables. L'analyse de Neysmith et Nichols révèle que les conditions de travail des prestateurs rémunérés sont sensiblement meilleures que celles des prestateurs non rémunérés. Toutefois, les prestateurs de soins rémunérés reçoivent un salaire peu élevé, peu de reconnaissance, ont très peu de possibilités d'avancement et peu de souplesse dans leurs conditions de travail. Les bénévoles semblent bénéficier des meilleures conditions de travail et jouir d'un statut élevé en dépit de l'absence d'une rémunération.

Les politiques gouvernementales vis-à-vis des prestateurs de soins à la famille se fondent sur la prémisse qu'une quantité minimale d'appui suffira pour permettre aux familles de continuer d'offrir des soins, mais ne tiennent pas compte de l'incidence à long terme des conditions de travail sur les prestateurs de soins. Neysmith et Nichols suggèrent que le gouvernement doit reconnaître ses obligations envers les aînés, peu importe leur situation de famille, en garantissant un éventail de services de base. Ces services donneraient aux familles un plus grand choix quant aux soins qu'elles désirent prodiguer et assureraient que tous les aînés puissent bénéficier de soins. Il s'agit d'une recommandation raisonnable qui met en lumière l'engagement que devrait prendre la société envers la prestation de soins aux aînés. Toutefois, une série de services de base ne suffirait pas à régler le problème de la prestation de soins. De nouvelles méthodes d'appui et le partage des responsabilités avec les prestateurs de soins au sein de la famille ainsi que l'amélioration de la situation des travailleurs en milieu familial sont également nécessaires. 
Les politiques actuelles sont limitées, car elles n'offrent pratiquement aucun moment de répit ni d'aide de divers groupes d'appui aux prestateurs de soins au sein de la famille. Les initiatives à venir doivent élargir l'étendue du soutien et comprendre des programmes en milieu de travail, des politiques offrant un congé à la famille, une compensation sur le plan fiscal et diverses autres formes de remboursements financiers pour les familles assurant des soins. La formation de prestateurs de soins sur la façon de prodiguer des soins ainsi qu'une formation professionnelle destinée aux personnes désirant retourner sur le marché du travail représentent des éléments importants d'un programme de soutien complet. Certaines précautions doivent être prises afin d'assurer que ces formes d'appui ne soient pas utilisées afin d'exploiter davantage les prestateurs de soins au sein de la famille. Certains programmes de remboursement destinés aux familles assurant la prestation de soins n'offrent actuellement que des subsides se situant au niveau du seuil de la pauvreté.

Les tâches du prestateur de soins à domicile comme celles de l'équipe de sontien doivent être conçues de façon à permettre une plus grande flexibilité, de sorte que les services puissent répondre adéquatement aux besoins de la clientèle. La formation offerte doit être plus approfondie et des postes plus élevés doivent être créés afin d'offrir des possibilités d'avancement. La plus importante amélioration à apporter à la situation de ces travailleurs est de reconnaître que les soins à domicile offrent de nombreux avantages sociaux au client qui vont au-delà des tâches ménagères. De fait, le but visé est de reconnaître que ces personnes occupent une place importante au sein de l'équipe des prestateurs de soins.

Le dilemme en matière de prestation de soins ne se situe donc pas seulement qu'au niveau des contraintes budgétaires au Canada. Le problème fondamental est la façon dont nous considérons les aînés au sein de notre société et dans quelle mesure les gouvernements reconnaissent leur responsabilité en matière de prestation de soins adéquats. Il est nécessaire de mettre de l'avant des méthodes innovatrices qui permettront d'offrir des soins adéquats à tous les aînés du Canada avec les fonds actuellement disponibles. Nous ne pouvons continuer de nous excuser ou de nous attendre à ce que les femmes et les familles comblent les lacunes et assurent la prestation de soins dans des conditions de travail déplorables. 